\title{
Weak monotonicity suffices for truthfulness on convex domains
}

\author{
Michael Saks, Lan Yu
}

January 10, 2005

\begin{abstract}
Weak monotonicity is a simple necessary condition for a social choice function to be implementable by a truthful mechanism. Roberts [10] showed that it is sufficient for all social choice functions whose domain is unrestricted. Lavi, Mu'alem and Nisan [6] proved the sufficiency of weak monotonicity for functions over order-based domains and Gui, Muller and Vohra [5] proved sufficiency for order-based domains with range constraints and for other special types of linear inequality constraints on the domain. Here we generalize these results by showing that weak monotonicity is sufficient for functions defined on any convex domain.
\end{abstract}

\section{Introduction}

Social choice theory centers around the general problem of selecting a single outcome out of a set $A$ of alternative outcomes based on the individual preferences of a set $P$ of players. A method for aggregating player preferences to select one outcome is called a social choice function. In this paper we assume that $A$ is finite and that each player's preference is expressed by a valuation function which assigns to each possible outcome a real number representing the "benefit" the player derives from that outcome. The ensemble of player valuation functions is viewed as a valuation matrix with rows indexed by players and columns by outcomes.

A major difficulty connected with social choice functions is that players can not be required to tell the truth about their preferences. Since each player seeks to maximize his own benefit, he may find it in his interest to misrepresent his valuation function. An important approach for dealing with this problem is to augment a given social choice function with a payment function, which assigns to each player a (positive or negative) payment as a function of all of the individual preferences. By carefully choosing the payment function, one can hope to entice each player to tell the truth. A social choice function augmented with a payment function is called a mechanism ${ }^{1}$ and the mechanism is said to implement the social choice function. A mechanism is truthful (or to be strategyproof or to have a dominant strategy) if each player's best strategy, knowing the preferences of the others, is always to declare his own true preferences. A social choice function is truthfully implementable, or truthful if it has a truthful implementation. (The property of truthful implementability is sometimes called dominant strategy incentive compatibility). This framework leads naturally to the question: which social choice functions are truthful?

This question is of the following general type: given a class of functions (here, social choice functions) and a property that holds for some of them (here, truthfulness), "characterize" the property. The definition of the property itself provides a characterization, so what more is needed? Here are some useful notions of characterization:

- Recognition algorithm. Give an algorithm which, given an appropriate representation of a function in the class, determines whether the function has the property.

\footnotetext{
${ }^{1}$ The usual definition of mechanism is more general than this; the mechanisms we consider here are usually called direct revelation mechanisms.
} 
- Parametric representation. Give an explicit parametrized family of functions and show that each function in the family has the property, and that every function with the property is in the family.

A third notion applies in the case of hereditary properties of functions. A function $g$ is a subfunction of function $f$, or $f$ contains $g$, if $g$ is obtained by restricting the domain of $f$ and a property $\mathcal{P}$ of functions is hereditary if it is preserved under taking subfunctions. Truthfulness is easily seen to be hereditary.

- Sets of obstructions. For a hereditary property $\mathcal{P}$, a function $g$ that does not have the property is an obstruction to the property in the sense that any function containing $g$ doesn't have the property. An obstruction is minimal if every proper subfunction has the property. A set of obstructions is complete if every function that does not have the property contains one of them as a subfunction. The set of all functions that don't satisfy $\mathcal{P}$ is a complete (but trivial and uninteresting) set of obstructions; one seeks a set of small (ideally, minimal) obstructions.

We are not aware of any work on recognition algorithms for the property of truthfulness, but there are significant results concerning parametric representations and obstruction characterizations of truthfulness. It turns out that the domain of the function, i.e., the set of allowed valuation matrices, is crucial. For functions with unrestricted domain, i.e., whose domain is the set of all real matrices, there are very good characterizations of truthfulness, but for general domains, the picture is far from complete. Typically, the domains of social choice functions are specified by a system of constraints. For example, an order constraint requires that one specified entry in some row be larger than another in the same row, a range constraint places an upper or lower bound on an entry, and a zero constraint forces an entry to be 0 . These are all examples of linear inequality constraints on the matrix entries.

Building on work of Roberts [10] and Bikhchandani, Chatterji and Sen [3], Lavi, Mu'alem and Nisan [6] defined a condition called weak monotonicity (W-MON). The definition of W-MON can be formulated in terms of obstructions: for some specified simple set $\mathcal{F}$ of functions each having domains of size 2, a function satisfies W-MON if it contains no function from $\mathcal{F}$. The functions in $\mathcal{F}$ are not truthful, and therefore $\mathrm{W}-\mathrm{MON}$ is a necessary condition for truthfulness. Lavi, Mu'alem and Nisan [6] showed that W-MON is also sufficient for truthfulness for social choice functions whose domain is order-based, i.e., defined by order constraints and zero constraints, and Gui, Muller and Vohra [5] extended this to other domains. The domain constraints considered in both papers are special cases of linear inequality constraints, and it is natural to ask whether W-MON is sufficient for any domain defined by such constraints. The main result of this paper is an affirmative answer to this question:

Theorem 1 For any social choice function with convex domain, weak monotonicity is necessary and sufficient for truthfulness.

Using the interpretation of weak monotonicity in terms of obstructions each having domain size 2 , this provides a complete set of minimal obstructions for truthfulness within the class of social choice functions with convex domains.

\section{$1.1 \quad$ Related work}

There is a simple and natural parametrized set of truthful social choice functions called affine maximizers. Roberts [10] showed that for functions with unrestricted domain, every truthful function is an affine maximizer, thus providing a parametrized representation for truthful functions with unrestricted domain. There are many known examples of truthful functions over restricted domains that are not affine maximizers (see [1], [2], [4], [6] and [7]). These examples have special structures and it seems plausible that there might be some mild restrictions on the class of all social choice functions such that all truthful functions obeying these restrictions are affine maximizers. Lavi, Mu'alem and 
Nisan [6] obtained a result in this direction by showing that for order-based domains, under certain technical assumptions, every truthful social choice function is "almost" an affine maximizer.

There are a number of results about truthfulness that can be viewed as providing an obstruction characterizations, although the notion of obstruction is not explicitly discussed.

For a player $i$, a set of valuation matrices is said to be $i$-local if all of the matrices in the set are identical except for row $i$. Call a social choice function $i$-local if its domain is $i$-local and call it local if it is $i$-local for some $i$. The following easily proved fact is used extensively in the literature:

Proposition 2 The social choice function $f$ is truthful if and only if every local subfunction of $f$ is truthful.

This implies that the set of all local non-truthful functions comprises a complete set of obstructions for truthfulness. This set is much smaller than the set of all non-truthful functions, but is still far from a minimal set of obstructions.

Rochet [11], Rozenshtrom [12] and Gui, Muller and Vohra [5] identified a necessary and sufficient condition for truthfulness (see lemma 3 below) called the nonnegative cycle property. This condition can be viewed as providing a minimal complete set of non-truthful functions. As is required by proposition 2, each function in the set is local. Furthermore it is one-to-one. In particular its domain has size at most the number of possible outcomes $|A|$.

As this complete set of obstructions consists of minimal non-truthful functions, this provides the optimal obstruction characterization of non-truthful functions within the class of all social choice functions. But by restricting attention to interesting subclasses of social choice functions, one may hope to get simpler sets of obstructions for truthfulness within that class.

The condition of weak monotonicity mentioned earlier can be defined by a set of obstructions, each of which is a local function of domain size exactly 2. Thus the results of Lavi, Mu'alem and Nisan [6], and of Gui, Muller and Vohra [5] give a very simple set of obstructions for truthfulness within certain subclasses of social choice functions. Theorem 1 extends these results to a much larger subclass of functions.

\subsection{Weak monotonicity and the nonnegative cycle property}

By proposition 2, a function is truthful if and only if each of its local subfunctions is truthful. Therefore, to get a set of obstructions for truthfulness, it suffices to obtain such a set for local functions.

The domain of an $i$-local function consists of matrices that are fixed on all rows but row $i$. Fix such a function $g$ and let $D \subseteq \mathbb{R}^{A}$ be the set of allowed choices for row $i$. We can associate $g$ with the function $f: D \longrightarrow A$ obtained by fixing the other rows as required for the domain of $g$. $f$ can be viewed as a social choice function having one player; we refer to such a function as a single player function.

Associated to any single player function $f$ with domain $D$ we define an edge-weighted directed graph $H_{f}$ whose vertex set is the image of $f$. For convenience, we assume that $f$ is surjective and so this image is $A$. For each $a, b \in A, x \in f^{-1}(a)$ there is an edge $e_{x}(a, b)$ from $a$ to $b$ with weight $x(a)-x(b)$. The weight of a set of edges is just the sum of the weights of the edges. We say that $f$ satisfies:

- the nonnegative cycle property if every directed cycle has nonnegative weight.

- the nonnegative two-cycle property if every directed cycle between two vertices has nonnegative weight.

We say a local function $g$ satisfies nonnegative cycle property/nonnegative two-cycle property if its associated single player function $f$ does.

The graph $H_{f}$ has a possibly infinite number of edges between any two vertices. We define $G_{f}$ to be the edge-weighted directed graph with exactly one edge from $a$ to $b$, whose weight $\delta_{a b}$ is the 
infimum (possibly $-\infty$ ) of all of the edge weights $e_{x}(a, b)$ for $x \in f^{-1}(a)$. It is easy to see that $H_{f}$ has the nonnegative cycle property/nonnegative two-cycle property if and only if $G_{f}$ does. $G_{f}$ is called the outcome graph of $f$.

The weak monotonicity property mentioned earlier can be defined for arbitrary social choice functions by the condition that every local subfunction satisfies the nonnegative two-cycle property. The following result was obtained by Rochet [11] in a slightly different form and rediscovered by Rozenshtrom [12] and Gui, Muller and Vohra [5]:

Lemma 3 A local social choice function is truthful if and only if it has the nonnegative cycle property. Thus a social choice function is truthful if and only if every local subfunction satisfies the nonnegative cycle property.

In light of this, our main result theorem 1 follows from:

Theorem 4 For any surjective single player function $f: D \longrightarrow A$ where $D$ is a convex subset of $\mathbb{R}^{A}$, the nonnegative two-cycle property implies the nonnegative cycle property.

This is the result we will prove.

\subsection{Overview of the proof of theorem 4}

Let $D \subseteq \mathbb{R}^{A}$ be convex and let $f: D \longrightarrow A$ be a single player function such that $G_{f}$ has no negative two-cycles. We want to conclude that $G_{f}$ has no negative cycles. For two vertices $a, b$, let $\delta_{a b}^{*}$ denote the minimum weight of any path from $a$ to $b$. Clearly $\delta_{a b}^{*} \leq \delta_{a b}$. Our proof shows that the $\delta^{*}$-weight of every cycle is exactly 0 , from which the main theorem follows.

The difficulty in the proof comes in the fact that there seems to be no direct way to compute $\delta^{*}$. We must proceed somewhat indirectly. Based on geometric considerations, we identify a subset of paths in $G_{f}$ called admissible paths and a subset of admissible paths called straight paths. We prove that for any two outcomes $a, b$, there is a straight path from $a$ to $b$ (lemma 8 and corollary 9 ), and all straight paths have the same weight, which we denote $\rho_{a b}$ (theorem 11). We show that $\rho_{a b} \leq \delta_{a b}$ (lemma 12) and that the $\rho$-weight of every cycle is 0 . The key step to this proof is showing that the $\rho$-weight of every directed triangle is 0 (lemma 15).

It turns out that $\rho$ is equal to $\delta^{*}$ (corollary 17 ), although this fact are not needed in the proof of the main theorem.

To expand on the above summary, we give the definitions of an admissible path and a straight path. These are somewhat technical and rely on the geometry of $f$. We first observe that, without loss of generality, we can assume that $D$ is (topologically) closed (section 2). In section 3 , for each $a \in A$, we define $D_{a}$ to be the closure of $f^{-1}(a)$. The negative two-cycle property and the fact that $D$ is closed and convex imply that each of these sets is closed and convex; their union is $D$ and their interiors are pairwise disjoint. We define an admissible path to be a sequence of outcomes $\left(a_{1}, \ldots, a_{k}\right)$ such that each of the sets $I_{j}=D_{a_{j}} \cap D_{a_{j+1}}$ is nonempty (section 4). An admissible path is straight if there is a straight line that meets one point from each of the sets $I_{1}, \ldots, I_{k-1}$ in order (section 5).

Finally, we mention the importance of convexity in the hypothesis of the main theorem. It is needed to show: (1) the existence of a straight path from $a$ to $b$ for all $a, b$. (2) the convexity of the sets $D_{a}$ and (3) that the $\rho$-weight of a directed triangle is 0 .

\section{Reduction to closed domain}

We first reduce the theorem to the case that $D$ is closed. Write $D^{C}$ for the closure of $D$. Since $A$ is finite, $D^{C}=\cup_{a \in A}\left(f^{-1}(a)\right)^{C}$. Thus for each $v \in D^{C}-D$, there is an $a(v)$ such that $v \in\left(f^{-1}(a)\right)^{C}$. Extend $f$ to the function $g$ on $D^{C}$ by defining $g(v)=a(v)$ for $v \in D^{C}-D$ and $g(v)=f(v)$ for 
$v \in D$. It is easy to check that $\delta_{a b}(g)=\delta_{a b}(f)$ for all $a, b \in A$ and therefore it suffices to show that the nonnegative two-cycle property for $g$ implies the nonnegative cycle property for $g$.

Henceforth we assume $D$ is convex and closed.

\section{A dissection of $D$}

In this section, we construct a family of closed convex sets $\left\{D_{a}: a \in A\right\}$ with disjoint interiors whose union is $D$ and satisfying $f^{-1}(a) \subseteq D_{a}$ for each $a \in A$.

Let $R_{a}=\left\{v: \forall b \in A, v(a)-v(b) \geq \delta_{a b}\right\} . R_{a}$ is a closed polyhedron containing $f^{-1}(a)$. The next proposition implies that any two of these polyhedra intersect only on their boundary.

Proposition 5 Let $a, b \in A$. If $v \in R_{a} \cap R_{b}$ then $v(a)-v(b)=\delta_{a b}=-\delta_{b a}$.

Proof: $v \in R_{a}$ implies $v(a)-v(b) \geq \delta_{a b}$ and $v \in R_{b}$ implies $v(b)-v(a) \geq \delta_{b a}$ which, by the nonnegative two-cycle property, implies $v(a)-v(b) \leq \delta_{a b}$. Thus $v(a)-v(b)=\delta_{a b}$ and by symmetry $v(b)-v(a)=\delta_{b a}$.

Finally, we restrict the collection of sets $\left\{R_{a}: a \in A\right\}$ to the domain $D$ by defining $D_{a}=R_{a} \cap D$ for each $a \in A$. Clearly, $D_{a}$ is closed and convex, and contains $f^{-1}(a)$. Therefore $\bigcup_{a \in A} D_{a}=D$. Also, by proposition 5 , any point $v$ in $D_{a} \cap D_{b}$ satisfies $v(a)-v(b)=\delta_{a b}=-\delta_{b a}$.

\section{Paths and $D$-sequences}

A path of size $k$ is a sequence $a=\left(a_{1}, \ldots, a_{k}\right)$ with each $a_{i} \in A$ (possibly with repetition). We call $a$ an $\left(a_{1}, a_{k}\right)$-path. For a path $a$, we write $|a|$ for the size of $a . a$ is simple if the $a_{i}$ 's are distinct.

For $b, c \in A$ we write $P_{b c}$ for the set of $(b, c)$-paths and $S P_{b c}$ for the set of simple $(b, c)$-paths. The $\delta$ cost of path $a$ is defined by $\delta(a)=\sum_{i=1}^{k-1} \delta_{a_{i} a_{i+1}}$.

A $D$-sequence of order $k$ is a sequence $u=\left(u_{0}, \ldots, u_{k}\right)$ with each $u_{i} \in D$ (possibly with repetition). We call $u$ a $\left(u_{0}, u_{k}\right)$-sequence. For a $D$-sequence $u$, we write $\operatorname{ord}(u)$ for the order of $u$. For $v, w \in D$ we write $S^{v w}$ for the set of $(v, w)$-sequences.

A compatible pair is a pair $(a, u)$ where $a$ is a path and $u$ is a $D$-sequence satisfying $\operatorname{ord}(u)=|a|$ and for each $i \in[k]$, both $u_{i-1}$ and $u_{i}$ belong to $D_{a_{i}}$.

We write $C(a)$ for the set of $D$-sequences $u$ that are compatible with $a$. We say that $a$ is admissible if $C(a)$ is nonempty. For $u \in C(a)$ we define $\Delta_{a}(u)=\sum_{i=1}^{|a|-1}\left(u_{i}\left(a_{i}\right)-u_{i}\left(a_{i+1}\right)\right)$.

For $v, w \in D$ and $b, c \in A$, we define $C_{b c}^{v w}$ to be the set of compatible pairs $(a, u)$ such that $a \in P_{b c}$ and $u \in S^{v w}$.

Proposition 6 For any compatible pair $(a, u), \Delta_{a}(u)=\delta(a)$.

Proof: $\quad$ Let $k=\operatorname{ord}(u)=|a|$. By the definition of a compatible pair, $u_{i} \in D_{a_{i}} \cap D_{a_{i+1}}$ for $i \in[k-1] \cdot u_{i}\left(a_{i}\right)-u_{i}\left(a_{i+1}\right)=\delta_{a_{i} a_{i+1}}$ from proposition 5. Therefore,

$$
\Delta_{a}(u)=\sum_{i=1}^{k-1}\left(u_{i}\left(a_{i}\right)-u_{i}\left(a_{i+1}\right)\right)=\sum_{i=1}^{k-1} \delta_{a_{i} a_{i+1}}=\delta(a) .
$$

Lemma 7 Let $b, c \in A$ and let $a, a^{\prime} \in P_{b c}$. If $C(a) \cap C\left(a^{\prime}\right) \neq \emptyset$ then $\delta(a)=\delta\left(a^{\prime}\right)$.

Proof: $\quad$ Let $u$ be a $D$-sequence in $C(a) \cap C\left(a^{\prime}\right)$. By proposition $6, \delta(a)=\Delta_{a}(u)$ and $\delta\left(a^{\prime}\right)=$ $\Delta_{a^{\prime}}(u)$, it suffices to show $\Delta_{a}(u)=\Delta_{a^{\prime}}(u)$. 
Let $k=\operatorname{ord}(u)=|a|=\left|a^{\prime}\right|$. Since

$$
\begin{gathered}
\Delta_{a}(u)=\sum_{i=1}^{k-1}\left(u_{i}\left(a_{i}\right)-u_{i}\left(a_{i+1}\right)\right) \\
=u_{1}\left(a_{1}\right)+\sum_{i=2}^{k-1}\left(u_{i}\left(a_{i}\right)-u_{i-1}\left(a_{i}\right)\right)-u_{k-1}\left(a_{k}\right) \\
=u_{1}(b)+\sum_{i=2}^{k-1}\left(u_{i}\left(a_{i}\right)-u_{i-1}\left(a_{i}\right)\right)-u_{k-1}(c), \\
\Delta_{a}(u)-\Delta_{a^{\prime}}(u)=\sum_{i=2}^{k-1}\left(\left(u_{i}\left(a_{i}\right)-u_{i-1}\left(a_{i}\right)\right)-\left(u_{i}\left(a_{i}^{\prime}\right)-u_{i-1}\left(a_{i}^{\prime}\right)\right)\right) \\
=\sum_{i=2}^{k-1}\left(\left(u_{i}\left(a_{i}\right)-u_{i}\left(a_{i}^{\prime}\right)\right)-\left(u_{i-1}\left(a_{i}\right)-u_{i-1}\left(a_{i}^{\prime}\right)\right)\right) .
\end{gathered}
$$

Noticing both $u_{i-1}$ and $u_{i}$ belong to $D_{a_{i}} \cap D_{a_{i}^{\prime}}$, we have by proposition 5

$$
u_{i-1}\left(a_{i}\right)-u_{i-1}\left(a_{i}^{\prime}\right)=\delta_{a_{i} a_{i}^{\prime}}=u_{i}\left(a_{i}\right)-u_{i}\left(a_{i}^{\prime}\right) .
$$

Hence $\Delta_{a}(u)-\Delta_{a^{\prime}}(u)=0$.

\section{$5 \quad$ Linear $D$-sequences and straight $A$-paths}

For $v, w \in D$ we write $\overline{v w}$ for the (closed) line segment joining $v$ and $w$.

A $D$-sequence $u$ of order $k$ is linear provided that there is a sequence of real numbers $0=\lambda_{0} \leq$ $\lambda_{1} \leq \ldots \leq \lambda_{k}=1$ such that $u_{i}=\left(1-\lambda_{i}\right) u_{0}+\lambda_{i} u_{k}$. In particular, each $u_{i}$ belongs to $\overline{u_{0} u_{k}}$. For $v, w \in D$ we write $L^{v w}$ for the set of linear $(v, w)$-sequences.

For $b, c \in A$ and $v, w \in D$ we write $L C_{b c}^{v w}$ for the set of compatible pairs $(a, u)$ such that $a \in P_{b c}$ and $u \in L^{v w}$.

For a path $a$, we write $L(a)$ for the set of linear sequences compatible with $a$. We say that $a$ is straight if $L(a) \neq \emptyset$.

Lemma 8 Let $b, c \in A$ and $v \in D_{b}, w \in D_{c}$. There is a simple path a and $D$-sequence $u$ such that $(a, u) \in L C_{b c}^{v w}$. Furthermore, for any such path $a, \delta(a) \leq v(b)-v(c)$.

Proof: By the convexity of $D$, any sequence of points on $\overline{v w}$ is a $D$-sequence.

If $b=c$, singleton path $a=(b)$ and $D$-sequence $u=(v, w)$ are obviously compatible. $\delta(a)=0=$ $v(b)-v(c)$.

So assume $b \neq c$. If $D_{b} \cap D_{c} \cap \overline{v w} \neq \emptyset$, we pick an arbitrary $x$ from this set and let $a=(b, c) \in$ $S P_{b c}, u=(v, x, w) \in L^{v w}$. Again it is easy to check the compatibility of $(a, u)$. Since $v \in D_{b}$, $v(b)-v(c) \geq \delta_{b c}=\delta(a)$.

For the remaining case $b \neq c$ and $D_{b} \cap D_{c} \cap \overline{v w}=\emptyset$, notice $v \neq w$ otherwise $v=w \in D_{b} \cap D_{c} \cap \overline{v w}$. So we can define $\lambda_{x}$ for every point $x$ on $\overline{v w}$ to be the unique number in $[0,1]$ such that $x=$ $\left(1-\lambda_{x}\right) v+\lambda_{x} w$. For convenience, we write $x \leq y$ for $\lambda_{x} \leq \lambda_{y}$.

Let $I_{a}=D_{a} \cap \overline{v w}$ for each $a \in A$. Since $D=\cup_{a \in A} D_{a}$, we have $\overline{v w}=\cup_{a \in A} I_{a}$. Moreover, by the convexity of $D_{a}$ and $\overline{v w}, I_{a}$ is a (possibly trivial) closed interval.

We begin by considering the case that $I_{b}$ and $I_{c}$ are each a single point, that is, $I_{b}=\{v\}$ and $I_{c}=\{w\}$.

Let $S$ be a minimal subset of $A$ satisfying $\cup_{s \in S} I_{s}=\overline{v w}$. For each $s \in S, I_{s}$ is maximal, i.e., not contained in any other $I_{t}$, for $t \in S$. In particular, the intervals $\left\{I_{s}: s \in S\right\}$ have all left endpoints 
distinct and all right endpoints distinct and the order of the left endpoints is the same as that of the right endpoints. Let $k=|S|+2$ and index $S$ as $a_{2}, \ldots, a_{k-1}$ in the order defined by the right endpoints. Denote the interval $I_{a_{i}}$ by $\left[l_{i}, r_{i}\right]$. Thus $l_{2}<l_{3}<\ldots<l_{k-1}, r_{2}<r_{3}<\ldots<r_{k-1}$ and the fact that these intervals cover $\overline{v w}$ implies $l_{2}=v, r_{k-1}=w$ and for all $2 \leq i \leq k-2, l_{i+1} \leq r_{i}$ which further implies $l_{i}<r_{i}$. Now we define the path $a=\left(a_{1}, a_{2}, \ldots, a_{k-1}, a_{k}\right)$ with $a_{1}=b, a_{k}=c$ and $a_{2}, a_{3}, \ldots, a_{k-1}$ as above. Define the linear sequence $u=\left(u_{0}, u_{1}, \ldots, u_{k}\right)$ by $u_{0}=u_{1}=v, u_{k}=w$ and for $2 \leq i \leq k-1, u_{i}=r_{i}$. It follows immediately that $(a, u) \in L C_{b c}^{v w}$. Neither $b$ nor $c$ is in $S$ since $l_{b}=r_{b}$ and $l_{c}=r_{c}$. Thus $a$ is simple.

Finally to show $\delta(a) \leq v(b)-v(c)$, we note

$$
v(b)-v(c)=v\left(a_{1}\right)-v\left(a_{k}\right)=\sum_{i=1}^{k-1}\left(v\left(a_{i}\right)-v\left(a_{i+1}\right)\right)
$$

and

$$
\delta(a)=\Delta_{a}(u)=\sum_{i=1}^{k-1}\left(u_{i}\left(a_{i}\right)-u_{i}\left(a_{i+1}\right)\right)=v\left(a_{1}\right)-v\left(a_{2}\right)+\sum_{i=2}^{k-1}\left(r_{i}\left(a_{i}\right)-r_{i}\left(a_{i+1}\right)\right) .
$$

Therefore it suffices to show $r_{i}\left(a_{i}\right)-r_{i}\left(a_{i+1}\right) \leq v\left(a_{i}\right)-v\left(a_{i+1}\right)$ for $2 \leq i \leq k-1$. For this it is enough to show that $z\left(a_{i}\right)-z\left(a_{i+1}\right)$ is a nonincreasing function of $z$ as $z$ moves from $v$ to $w$ along $\overline{v w}$. This follows from:

Fact 1 Let $\overline{v w}$ be a line segment in $D$ and let $d, e \in A$. The function $z(d)-z(e)$ is a linear function of $z$ and hence is monotone as $z$ moves along the segment from $v$ to $w$. Furthermore, if there are points $x, y$ on $\overline{v w}$ with $x<y, x \in D_{d}$ and $y \in D_{e}$ then $x(d)-x(e) \geq \delta_{d e} \geq-\delta_{e d} \geq-(y(e)-y(d))$. Thus $z(d)-z(e)$ is monotonically nonincreasing as $z$ moves along $\overline{v w}$ from $v$ to $w$.

Applying this fact with $d=a_{i}, e=a_{i+1}, x=l_{i}$ and $y=r_{i}$ gives the desired conclusion. This completes the proof for the case that $I_{b}=\{v\}$ and $I_{c}=\{w\}$.

For general $I_{b}, I_{c}, r_{b}<l_{c}$ otherwise $D_{b} \cap D_{c} \cap \overline{v w}=I_{b} \cap I_{c} \neq \emptyset$. Let $v^{\prime}=r_{b}$ and $w^{\prime}=l_{c}$. Clearly we can apply the above conclusion to $v^{\prime} \in D_{b}, w^{\prime} \in D_{c}$ and get a compatible pair $\left(a, u^{\prime}\right) \in L C_{b c}^{v^{\prime} w^{\prime}}$ with $a$ simple and $\delta(a) \leq v^{\prime}(b)-v^{\prime}(c)$. Define the linear sequence $u$ by $u_{0}=v, u_{k}=w$ and $u_{i}=u_{i}^{\prime}$ for $i \in[k-1] .(a, u) \in L C_{b c}^{v w}$ is evident. Moreover, applying the above fact with $d=b, e=c, x=v$ and $y=w$, we get $v(b)-v(c) \geq v^{\prime}(b)-v^{\prime}(c) \geq \delta(a)$.

Corollary 9 For any $b, c \in A$ there is a straight $(b, c)$-path.

The main result of this section (theorem 11) says that for any $b, c \in A$, every straight $(b, c)$-path has the same $\delta$-cost. First we need:

Lemma 10 For $b, c \in A$, there is a function $\rho_{b c}: D_{b} \times D_{c} \longrightarrow \mathbb{R}$ satisfying that for any $(a, u) \in$ $L C_{b c}^{v w}, \delta(a)=\rho_{b c}(v, w)$.

Proof: Let $\left(a^{\prime}, u^{\prime}\right),\left(a^{\prime \prime}, u^{\prime \prime}\right) \in L C_{b c}^{v w}$. It suffices to show $\delta(a)=\delta\left(a^{\prime}\right)$. To do this we construct a linear $(v, w)$-sequence $u$ and paths $a^{*}, a^{* *} \in P_{b c}$, both compatible with $u$, satisfying $\delta\left(a^{*}\right)=\delta\left(a^{\prime}\right)$ and $\delta\left(a^{* *}\right)=\delta\left(a^{\prime \prime}\right)$. Lemma 7 implies $\delta\left(a^{*}\right)=\delta\left(a^{* *}\right)$, which will complete the proof.

Let $\left|a^{\prime}\right|=\operatorname{ord}\left(u^{\prime}\right)=k$ and $\left|a^{\prime \prime}\right|=\operatorname{ord}\left(u^{\prime \prime}\right)=l$. We select $u$ to be any linear $(v, w)$-sequence $\left(u_{0}, u_{1}, \ldots, u_{t}\right)$ such that $u^{\prime}$ and $u^{\prime \prime}$ are both subsequences of $u$, i.e., there are indices $0=i_{0}<$ $i_{1}<\cdots<i_{k}=t$ and $0=j_{0}<j_{1}<\cdots<j_{l}=t$ such that $u^{\prime}=\left(u_{i_{0}}, u_{i_{1}}, \ldots, u_{i_{k}}\right)$ and $u^{\prime \prime}=$ $\left(u_{j_{0}}, u_{j_{1}}, \ldots, u_{j_{l}}\right)$. We now construct a $(b, c)$-path $a^{*}$ compatible with $u$ such that $\delta\left(a^{*}\right)=\delta\left(a^{\prime}\right)$. (An analogous construction gives $a^{* *}$ compatible with $u$ such that $\delta\left(a^{* *}\right)=\delta\left(a^{\prime \prime}\right)$.) This will complete the proof.

$a^{*}$ is defined as follows: for $1 \leq j \leq t, a_{j}^{*}=a_{r}^{\prime}$ where $r$ is the unique index satisfying $i_{r-1}<j \leq i_{r}$. Since both $u_{i_{r-1}}=u_{r-1}^{\prime}$ and $u_{i_{r}}=u_{r}^{\prime}$ belong to $D_{a_{r}^{\prime}}, u_{j} \in D_{a_{r}^{\prime}}$ for $i_{r-1} \leq j \leq i_{r}$ by the convexity 
of $D_{a_{r}^{\prime}}$. The compatibility of $\left(a^{*}, u\right)$ follows immediately. Clearly, $a_{1}^{*}=a_{1}^{\prime}=b$ and $a_{t}^{*}=a_{k}^{\prime}=c$, so $a^{*} \in P_{b c}$. Furthermore, as $\delta_{a_{j}^{*} a_{j+1}^{*}}=\delta_{a_{r}^{\prime} a_{r}^{\prime}}=0$ for each $r \in[k], i_{r-1}<j<i_{r}$,

$$
\delta\left(a^{*}\right)=\sum_{r=1}^{k-1} \delta_{a_{i_{r}}^{*} a_{i_{r+1}}^{*}}=\sum_{r=1}^{k-1} \delta_{a_{r}^{\prime} a_{r+1}^{\prime}}=\delta\left(a^{\prime}\right) .
$$

We are now ready for the main theorem of the section

Theorem $11 \rho_{b c}$ is a constant map on $D_{b} \times D_{c}$. Thus for any $b, c \in A$, every straight $(b, c)$-path has the same $\delta$-cost.

Proof: For a path $a,(v, w)$ is compatible with $a$ if there is a linear $(v, w)$-sequence compatible with $a$. We write $C P(a)$ for the set of pairs $(v, w)$ compatible with $a$. $\rho_{b c}$ is constant on $C P(a)$ because for each $(v, w) \in C P(a), \rho_{b c}(v, w)=\delta(a)$. By lemma 8, we also have $\bigcup_{a \in S P_{b c}} C P(a)=$ $D_{b} \times D_{c}$. Since $A$ is finite, $S P_{b c}$, the set of simple paths from $b$ to $c$, is finite as well.

Next we prove that for any path $a, C P(a)$ is closed.

Let $\left(\left(v^{n}, w^{n}\right): n \in \mathbb{N}\right)$ be a convergent sequence of points in $C P(a)$ and let $(v, w)$ be the limit. We want to show that $(v, w) \in C P(a)$. For each $n \in \mathbb{N}$, since $\left(v^{n}, w^{n}\right) \in C P(a)$, there is a linear $\left(v^{n}, w^{n}\right)$-sequence $u^{n}$ compatible with $a$, ie, there are $0=\lambda_{0}^{n} \leq \lambda_{1}^{n} \leq \ldots \leq \lambda_{k}^{n}=1(k=|a|)$ such that $u_{j}^{n}=\left(1-\lambda_{j}^{n}\right) v^{n}+\lambda_{j}^{n} w^{n}(j=0,1, \ldots, k)$. Since for each $n, \lambda^{n}=\left(\lambda_{0}^{n}, \lambda_{1}^{n}, \ldots, \lambda_{k}^{n}\right)$ belongs to the closed bounded set $[0,1]^{k+1}$ we can choose an infinite subset $I \subseteq \mathbb{N}$ such that the sequence $\left(\lambda^{n}: n \in I\right)$ converges. Let $\lambda=\left(\lambda_{0}, \lambda_{1}, \ldots, \lambda_{k}\right)$ be the limit. Clearly $0=\lambda_{0} \leq \lambda_{1} \leq \cdots \leq \lambda_{k}=1$.

Define the linear $(v, w)$-sequence $u$ by $u_{j}=\left(1-\lambda_{j}\right) v+\lambda_{j} w(j=0,1, \ldots, k)$. Then for each $j \in\{0, \ldots, k\}, u_{j}$ is the limit of the sequence $\left(u_{j}^{n}: n \in I\right)$. For $j>0$, each $u_{j}^{n}$ belongs to the closed set $D_{a_{j}}$, so $u_{j} \in D_{a_{j}}$. Similarly, for $j<k$ each $u_{j}^{n}$ belongs to the closed set $D_{a_{j+1}}$, so $u_{j} \in D_{a_{j+1}}$. Hence $(a, u)$ is compatible, implying $(v, w) \in C P(a)$.

Now we have $D_{b} \times D_{c}$ covered by finitely many closed subsets on each of them $\rho_{b c}$ is a constant.

Suppose for contradiction that there are $(v, w),\left(v^{\prime}, w^{\prime}\right) \in D_{b} \times D_{c}$ such that $\rho_{b c}(v, w) \neq \rho_{b c}\left(v^{\prime}, w^{\prime}\right)$. $L=\left\{\left((1-\lambda) v+\lambda v^{\prime},(1-\lambda) w+\lambda w^{\prime}\right): \lambda \in[0,1]\right\}$ is a line segment in $D_{b} \times D_{c}$ by the convexity of $D_{b}, D_{c}$. Let $L_{1}=\left\{(x, y) \in L: \rho_{b c}(x, y)=\rho_{b c}(v, w)\right\}$ and $L_{2}=L-L_{1}$. Clearly $(v, w) \in L_{1},\left(v^{\prime}, w^{\prime}\right) \in L_{2}$. Let $P=\left\{a \in S P_{b c}: \delta(a)=\rho_{b c}(v, w)\right\} . L_{1}=\left(\bigcup_{a \in P} C P(a)\right) \cap L, L_{2}=\left(\bigcup_{a \in S P_{b c}-P} C P(a)\right) \cap L$ are closed by the finiteness of $P$. This is a contradiction, since it is well known (and easy to prove) that a line segment can not be expressed as the disjoint union of two nonempty closed sets.

\section{Proof of Theorem 4}

Lemma $12 \rho_{b c} \leq \delta_{b c}$ for all $b, c \in A$.

Proof: For contradiction, suppose $\rho_{b c}-\delta_{b c}=\epsilon>0$. By the definition of $\delta_{b c}$, there exists $v \in f^{-1}(b) \subseteq D_{b}$ with $v(b)-v(c)<\delta_{b c}+\epsilon=\rho_{b c}$. Pick an arbitrary $w \in D_{c}$. By lemma 8, there is a compatible pair $(a, u) \in L C_{b c}^{v w}$ with $\delta(a) \leq v(b)-v(c)$. Therefore, $a$ is a straight $(b, c)$-path, $\rho_{b c}=\delta(a) \leq v(b)-v(c)$, leading to a contradiction.

Define another edge-weighted complete directed graph $G_{f}^{\prime}$ on vertex set $A$ where the weight of $\operatorname{arc}(a, b)$ is $\rho_{a b}$. Immediately from lemma 12 , the weight of every directed cycle in $G_{f}$ is bounded below by its weight in $G_{f}^{\prime}$. To prove theorem 1, it suffices to show the zero cycle property of $G_{f}^{\prime}$, ie, every directed cycle has weight zero. We begin by considering two-cycles.

Lemma $13 \rho_{b c}+\rho_{c b}=0$ for all $b, c \in A$.

Proof: Let $a$ be a straight $(b, c)$-path compatible with linear sequence $u$. let $a^{\prime}$ be the reverse of $a$ and $u^{\prime}$ the reverse of $u$. Obviously, $\left(a^{\prime}, u^{\prime}\right)$ is compatible as well and thus $a^{\prime}$ is a straight $(c, b)$-path. Therefore, 


$$
\rho_{b c}+\rho_{c b}=\delta(a)+\delta\left(a^{\prime}\right)=\sum_{i=1}^{k-1} \delta_{a_{i} a_{i+1}}+\sum_{i=1}^{k-1} \delta_{a_{i+1} a_{i}}=\sum_{i=1}^{k-1}\left(\delta_{a_{i} a_{i+1}}+\delta_{a_{i+1} a_{i}}\right)=0,
$$

where the final equality uses Proposition 5.

Lemma 14 If there are collinear points $u \in D_{a}, v \in D_{b}, w \in D_{c}(a, b, c \in A), \rho_{a b}+\rho_{b c}=\rho_{a c}$.

Proof: First, we prove for the case where $v$ is between $u$ and $w$. From lemma 8, there are compatible pairs $\left(a^{\prime}, u^{\prime}\right) \in L C_{a b}^{u v},\left(a^{\prime \prime}, u^{\prime \prime}\right) \in L C_{b c}^{v w}$. Let $\left|a^{\prime}\right|=\operatorname{ord}\left(u^{\prime}\right)=k$ and $\left|a^{\prime \prime}\right|=\operatorname{ord}\left(u^{\prime \prime}\right)=l$. We paste $a^{\prime}$ and $a^{\prime \prime}$ together as

$$
a^{\prime \prime \prime}=\left(a=a_{1}^{\prime}, a_{2}^{\prime}, \ldots, a_{k-1}^{\prime}, a_{k}^{\prime}, a_{1}^{\prime \prime}, \ldots, a_{l}^{\prime \prime}=c\right),
$$

$u^{\prime}$ and $u^{\prime \prime}$ as

$$
u^{\prime \prime \prime}=\left(u=u_{0}^{\prime}, u_{1}^{\prime}, \ldots, u_{k}^{\prime}=v=u_{0}^{\prime \prime}, u_{1}^{\prime \prime}, \ldots, u_{l}^{\prime \prime}=w\right) .
$$

Clearly $\left(a^{\prime \prime \prime}, u^{\prime \prime \prime}\right) \in L C_{a c}^{u w}$ and

$$
\delta\left(a^{\prime \prime \prime}\right)=\sum_{i=1}^{k-1} \delta_{a_{i}^{\prime} a_{i+1}^{\prime}}+\delta_{a_{k}^{\prime} a_{1}^{\prime \prime}}+\sum_{i=1}^{l-1} \delta_{a_{i}^{\prime \prime} a_{i+1}^{\prime \prime}}=\delta\left(a^{\prime}\right)+\delta_{b b}+\delta\left(a^{\prime \prime}\right)=\delta\left(a^{\prime}\right)+\delta\left(a^{\prime \prime}\right) .
$$

Therefore, $\rho_{a c}=\delta\left(a^{\prime \prime \prime}\right)=\delta\left(a^{\prime}\right)+\delta\left(a^{\prime \prime}\right)=\rho_{a b}+\rho_{b c}$.

Now suppose $w$ is between $u$ and $v$. By the above argument, we have $\rho_{a c}+\rho_{c b}=\rho_{a b}$ and by lemma $13, \rho_{a b}+\rho_{b c}=\rho_{a b}-\rho_{c b}=\rho_{a c}$.

The case that $u$ is between $v$ and $w$ is similar.

Lemma $15 \rho_{a b}+\rho_{b c} \geq \rho_{a c}$ for all $a, b, c \in A$.

Proof: $\quad$ Let $S=\left\{(a, b, c): \rho_{a b}+\rho_{b c}<\rho_{a c}\right\}$ and for contradiction, suppose $S \neq \emptyset$. $S$ is finite. For each $a \in A$, choose $v_{a} \in D_{a}$ arbitrarily and let $T$ be the convex hull of $\left\{v_{a}: a \in A\right\}$. For each $(a, b, c) \in S$, let $R_{a b c}=D_{a} \times D_{b} \times D_{c} \cap T 3$. Clearly, each $R_{a b c}$ is nonempty and compact. Moreover, by lemma 14 , no $(u, v, w) \in R_{a b c}$ is collinear.

Define $f: D 3 \rightarrow \mathbb{R}$ by $f(u, v, w)=|v-u|+|w-v|+|u-w|$. For $(a, b, c) \in S$, the restriction of $f$ to the compact set $R_{a b c}$ attains a minimum $m(a, b, c)$ at some point $(u, v, w) \in R_{a b c}$ by the continuity of $f$, i.e., there exists a triangle $\Delta u v w$ of minimum perimeter within $T$ with $u \in D_{a}, v \in D_{b}, w \in D_{c}$.

Choose $\left(a^{*}, b^{*}, c^{*}\right) \in S$ so that $m\left(a^{*}, b^{*}, c^{*}\right)$ is minimum and let $\left(u^{*}, v^{*}, w^{*}\right) \in R_{a^{*} b^{*} c^{*}}$ be a triple achieving it. Pick an arbitrary point $p$ in the interior of $\Delta u^{*} v^{*} w^{*}$. By the convexity of domain $D$, there is $d \in A$ such that $p \in D_{d}$.

Consider triangles $\Delta u^{*} p w^{*}, \Delta w^{*} p v^{*}$ and $\Delta v^{*} p u^{*}$. Since each of them has perimeter less than that of $\Delta u^{*} v^{*} w^{*}$ and all three triangles are contained in $T$, by the minimality of $\Delta u^{*} v^{*} w^{*},\left(a^{*}, d, c^{*}\right)$, $\left(c^{*}, d, b^{*}\right),\left(b^{*}, d, a^{*}\right) \notin S$. Thus

$$
\begin{aligned}
& \rho_{a^{*} c^{*}} \leq \rho_{a^{*} d}+\rho_{d c^{*}}, \\
& \rho_{c^{*} b^{*}} \leq \rho_{c^{*} d}+\rho_{d b^{*}}, \\
& \rho_{b^{*} a^{*}} \leq \rho_{b^{*} d}+\rho_{d a^{*}} .
\end{aligned}
$$

Summing up the three inequalities,

$$
\rho_{a^{*} c^{*}}+\rho_{c^{*} b^{*}}+\rho_{b^{*} a^{*}} \leq \rho_{a^{*} d}+\rho_{d c^{*}}+\rho_{c^{*} d}+\rho_{d b^{*}}+\rho_{b^{*} d}+\rho_{d a^{*}}=0
$$

which yields a contradiction

$$
\rho_{a^{*} b^{*}}+\rho_{b^{*} c^{*}}=-\rho_{b^{*} a^{*}}-\rho_{c^{*} b^{*}} \geq \rho_{a^{*} c^{*}} .
$$


Triangle equality $\rho_{a b}+\rho_{b c}=\rho_{a c}$ follows since for all $a, b, c \in A$, applying lemma 15 to $a, c, b$ gives $\rho_{a c}+\rho_{c b} \geq \rho_{a b}$, implying the other direction $\rho_{a b}+\rho_{b c}=\rho_{a b}-\rho_{c b} \leq \rho_{a c}$.

With zero two-cycle property and triangle equality, we now complete the proof by showing the zero cycle property of $G_{f}^{\prime}$. By inductively applying triangle equality, we have $\sum_{i=1}^{k-1} \rho_{a_{i} a_{i+1}}=\rho_{a_{1} a_{k}}$. Therefore, the weight of a directed cycle $C=a_{1} a_{2} \ldots a_{k} a_{1}$ is

$$
\sum_{i=1}^{k-1} \rho_{a_{i} a_{i+1}}+\rho_{a_{k} a_{1}}=\rho_{a_{1} a_{k}}+\rho_{a_{k} a_{1}}=0 .
$$

As final remarks, we note that our result implies the following strengthenings of theorem 11:

Corollary 16 For any $b, c \in A$, every admissible $(b, c)$-path has the same $\delta$-cost $\rho_{b c}$.

Proof: First notice that for any $b, c \in A$, if $D_{b} \cap D_{c} \neq \emptyset, \delta_{b c}=\rho_{b c}$. To see this, pick $v \in D_{b} \cap D_{c}$ arbitrarily. Obviously, path $a=(b, c)$ is compatible with linear sequence $u=(v, v, v)$ and is thus a straight $(b, c)$-path. Hence $\rho_{b c}=\delta(a)=\delta_{b c}$.

Now for any $b, c \in A$ and any $(b, c)$-path $a$ with $C(a) \neq \emptyset$, let $u \in C(a)$. Since $u_{i} \in D_{a_{i}} \cap D_{a_{i+1}}$ for $i \in[|a|-1]$,

$$
\delta(a)=\sum_{i=1}^{|a|-1} \delta_{a_{i} a_{i+1}}=\sum_{i=1}^{|a|-1} \rho_{a_{i} a_{i+1}}=\rho_{a_{1} a_{|a|}}=\rho_{b c} .
$$

Corollary 17 For any $b, c \in A, \rho_{b c}$ is the minimum $\delta$-cost over all $(b, c)$-paths.

Proof: ¿From lemma 12, $\rho_{b c}$ is a lower bound for the $\delta$-cost of every $(b, c)$-path. On the other hand, there exists a straight $(b, c)$-path of cost $\rho_{b c}$ by corollary 9 and theorem 11 .

\section{Acknowledgments}

We thank Ron Lavi for helpful comments and discussions.

\section{References}

[1] Aaron Archer and Eva Tardos. Truthful mechanisms for one-parameter agents. Proc. of the 42nd Annual Symposium on Foundations of Computer Science (FOCS'01), 2001.

[2] Yair Bartal, Rica Gonen, and Noam Nisan. Incentive compatible multi-unit combinatorial auctions. Proc. of the 9th Conference on Theoretical Aspects of Rationality and Knowledge (TARK'03), 2003.

[3] Sushil Bikhchandani, Shurojit Chatterji and Arunava Sen. Incentive compatibility in multi-unit auctions, 2004. Working paper.

[4] Andrew Goldberg, Jason Hartline, Anna Karlin, Michael Saks and Andrew Wright. Competitive Auctions, 2003.

[5] Hongwei Gui, Rudolf Muller and Rakesh Vohra. Dominant strategy mechanisms with multidimensional types, 2004. Working paper.

[6] Ron Lavi, Ahuva Mu'alem and Noam Nisan. Towards a characterization of truthful combinatorial auctions. Proc. of the 44th Annual Symposium on Foundations of Computer Science (FOCS'03), 2003. 
[7] Daniel Lehmann, Liadan O'Callaghan and Yoav Shoham. Truth revelation in approximately efficient combinatorial auctions. Journal of the ACM, 49(5):577-602, 2002.

[8] Andreu Mas-Colell, Michael Whinston and Jerry Green. Microeconomic Theory. Oxford University Press, 1995.

[9] Noam Nisan. Algorithms for selfish agents. Proc. of the 16th Symposium on Theoretical Aspects of Computer Science (STACS'99), 1999.

[10] Kevin Roberts. The characterization of implementable choice rules. Aggregation and Revelation of Preferences, J-J. Laffont (ed.), North Holland Publishing Company.

[11] Jean-Charles Rochet. A necessary and sufficient condition for rationalizability in a quasi-linear context. Journal of Mathematical Economics, 16:191-200, 1987.

[12] Irit Rozenshtrom. Dominant strategy implementation with quasi-linear preferneces, 1999. Master's thesis, Dept. of Economics, The Hebrew University, Jerusalem, Israel. 